\title{
Hershberger Assays for Di-2-ethylhexyl Phthalate and Its Substitute Candidates
}

\author{
Hee-Su Kim¹, Yong-Pil Cheon ${ }^{2}$, and ${ }^{\dagger}$ Sung-Ho Lee ${ }^{1}$ \\ ${ }^{1}$ Dept. of Biotechnology, Sangmyung University, Seoul 03016, Korea \\ ${ }^{2}$ Division of Developmental Biology and Physiology, School of Biological Sciences and Chemistry, \\ Sungshin University, Seoul 02844, Korea
}

\begin{abstract}
In the present study, we employed Hershberger assay to determine possible androgenic or antiandrogenic activities of three di-2-ethylhexyl phthalate (DEHP) substitute candidates. The assay was carried out using immature castrated Sprague-Dawley male rats. After 7 days of the surgery, testosterone propionate (TP, $0.4 \mathrm{mg} / \mathrm{kg} / \mathrm{day}$ ) and test materials (low dose, $40 \mathrm{mg} / \mathrm{kg} /$ day; high dose, $400 \mathrm{mg} / \mathrm{kg} /$ day) were administered for 10 consecutive days by subcutaneous (s.c.) injection and oral gavage, respectively. Test materials were DEHP, 2-ethylhexyl oleate (IOO), 2-ethylhexyl stearate (IOS) and triethyl 2-acetylcitrate (ATEC). The rats were necropsied, and then the weights of five androgen-dependent tissues [ventral prostate, seminal vesicle, coagulating glands, levator ani-bulbocavernosus (LABC) muscle, paired Cowper's glands, and glans penis] and four androgen-insensitive tissues (kidney, adrenal glands, spleen and liver) were measured. All test materials including DEHP did not exhibit any androgenic activity in the assay. On the contrary, antiandrogen-like activities were found in all test groups, and the order of the intensity was ATEC $<$ DEHP $<$ ISO $<$ IOO in the five androgen-sensitive tissues. There was no statistical difference between low dose treatment and high dose treatment of all replacement candidate groups. In DEHP groups, high dose treatment exhibited significant weight gains in LABC and Glan Penis. There was no statistical difference in androgen-insensitive tissue measurements. Since the effects of ATEC treatment on the accessory sex organs were much less or not present at all when compared to those of DEHP, ATEC could be a strong candidate to replace DEHP. IOO treatment brought most severe weight reduction in all of androgen-sensitive tissues, so this material should be excluded for further screening of DEHP substitute selection.
\end{abstract}

Key words : Rat, Hershberger assay, Di-2-ethylhexyl phthalate (DEHP), Substitute

\section{INTRODUCTION}

Phthalates or phthalate esters, are esters of phthalic acid, and they are mainly used as plasticizers, i.e., substances added to plastics to increase their flexibility, transparency, durability, and longevity (Cao, 2009). Since the plasticized materials are indispensable in everyday lives, the majority of people in modernized nations are exposed to some level of phthalates (Meeker et al., 2008). For example, multiple phthalates metabolites were detected in human urine from several Asian countries including South Korea (Guo et al., 2011).

\footnotetext{
Manuscript received March 17, 2018, Received in revised form March 22, 2018, Accepted March 25, 2018

${ }^{\dagger}$ Corresponding Author : Sung-Ho Lee, Ph.D., Dept. of Biotechnology, Sangmyung University, Seoul 03016, Korea. Tel: +82-2-2287-5139, Fax: +82-22287-0070, E-mail: shlee@smu.ac.kr

This is an Open Access article distributed under the terms of the Creative Commons Attribution Non-Commercial License (http:// creative-commons.org/licenses/by-nc/3.0) which permits unrestricted non-commercial use, distribution, and reproduction in any medium, provided the original work is properly cited.
} 
Di-2-ethylhexyl phthalate (DEHP) is the most common member of the phthalates family, and along with other phthalates, DEHP is known to cause endocrine disruption. Indeed, numerous studies have demonstrated a potential for DEHP to adversely impact the male and female reproductive systems such are abnormal reproductive tract formation, lower fertility, pregnancy loss, abnormal puberty onset, endometriosis, and abnormal breast development (Gray et al., 2000; Lyche et al., 2009; MartinoAndrade \& Chahoud, 2010; Huang et al., 2012; Kay et al., 2013).

Due to many detrimental effects of DEHP, particularly on the fetus and newborn, the task making plastic products with DEHP alternatives becomes much more urgent. Although there are some biological alternatives on the market, the common problem is that they are typically expensive and not compatible as a primary plasticizer. Concurrently, the potential negative effects of the candidates should be accurately and efficiently screened. Previously we evaluated the two bisphenol-A (BPA) substitute candidates whether they have androgenic or antiandrogenic activity using Hershberger assay, and found isosorbide could be a safer candidate while cyclohexanedimethanol exerted more detrimental effects (Kim et al., 2017). In the present study, we employed the identical in vivo animal model system to determine possible androgenic or antiandrogenic activities of three DEHP substitute candidates.

\section{MATERIALS AND METHODS}

\section{Chemicals}

Testosterone propionate (TP), bis(2-ethylhexyl) phthalate (DEHP, CAS number 117-81-7), triethyl 2-acetylcitrate (ATEC, CAS number 77-89-4) and corn oil were purchased from Sigma Chemical Co. (St. Louis, MO, USA). 2-ethylhexyl oleate (IOO, CAS number 26399-02-0) and 2-ethylhexyl stearate (IOS, CAS number 22047-49-0) were donated from SFC Co. (Seoul, Korea). All substances were stored in glass containers at room temperature (Table 1).

\section{Animals and ethics approval}

Male Sprague-Dawley rats (28 days old) were obtained from DBL (Chungcheongbuk-do, Korea) and reared in Sangmyung University animal facility under specific pathogen free (SPF)-conditions of 12-h light/dark cycle (lights on at 07:00 h) and constant temperature of $22 \pm 1^{\circ} \mathrm{C}$. Rats had free access to normal chow (Purina, Seoul, Korea) and tap water ad libitum. All procedures used were approved by the Animal Care and Use Committee (2017-02-03) at Sangmyung University. All the animals were handled in accordance with the guidelines for animal experiments of the Association for Assessment and Accreditation of Laboratory Animal Care (AAALAC).

\section{Surgery and test substance administrations}

Rats were castrated (ORX) on postnatal day (PND) 42 under anesthesia by placing an incision in the scrotum and removing both testes and epididymides with a ligation of the blood vessels and seminal ducts. The animals were allowed to recover from castration for 7 days, and then animals were randomly assigned to different groups in terms of their body weights on PND $49(\mathrm{n}=6)$. TP was daily administered for 10 consecutive days by subcutaneous (s.c.) injection at a dose of $0.4 \mathrm{mg} / \mathrm{kg}$ bw/day (dissolved in $0.5 \mathrm{~mL}$ corn oil). The route of administration for the test substances (low dose, $40 \mathrm{mg} / 5 \mathrm{~mL} / \mathrm{kg}$ bw; high dose, 400 $\mathrm{mg} / 5 \mathrm{~mL} / \mathrm{kg}$ bw) was via oral gavage.

For the androgenic activity test, the vehicle group (ORX) was the negative control, and the TP-treated group (ORX+ TP) was the positive control. The weights of the tissues were compared to the controls for statistically significant changes. Two dose groups of the test substance plus positive and vehicle (negative) controls were normally sufficient to determine if a chemical is an androgen agonist, and this design was therefore preferred for animal welfare reasons (EPA, 2011). 
Table 1. Chemical structure of DEHP and its substitute candidates

\begin{tabular}{ccc}
\hline \hline Component & CAS number & Chemical structure \\
DEHP \\
(bis(2-ethylhexyl) phthalate)
\end{tabular}

IOO

(2-ethylhexyl oleate)

26399-02-0

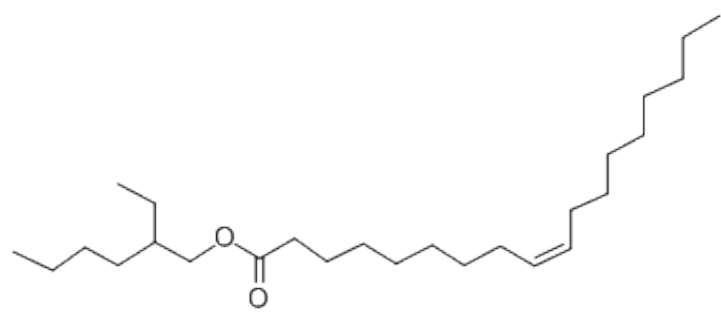

IOS

(2-ethylhexyl stearate)

$22047-49-0$

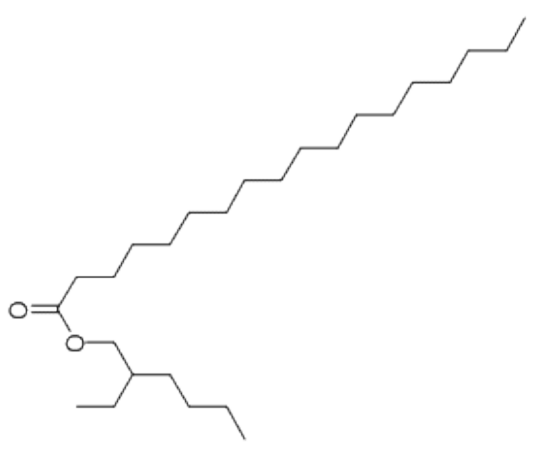

ATEC

(triethyl 2-acetylcitrate)

$77-89-4$

\section{Sample collections}

Approximately 24 hours after the final administration of the test substance, the rats were necropsied according to standard laboratory procedures. As critical indices, the weights of five androgen-dependent tissues [ventral prostate, seminal vesicle, coagulating glands, levator anibulbocavernosus (LABC) muscle, paired Cowper's glands, and glans penis] were measured. The weights of optional tissues (kidney, adrenal glands, spleen and liver) were also measured.

\section{Statistical analysis}

All values are expressed as mean S.E. $(n=6)$. Absolute tissues weights were analyzed using analysis of covariance (ANOVA) or Student's $t$-test. Data were expressed as mean $\pm \mathrm{SE}$, and $p$-value $<0.05$ denoted the statistically sig- 
nificant difference.

\section{RESULTS}

No abnormal clinical wounds or body weight changes were observed in the rats administered with the test chemicals.

\section{Effects on androgen-dependent tissues}

The effects of DEHP and replacement candidates on changes in body weights and androgen-dependent tissues (seminal vesicles, ventral prostate, LABC, paired Cowper's glands, and glans penis) weights in castrated male rats are shown in Tables 2. In all organs, the most severe weight loss was commonly found in IOO group.

\section{1) Body weights}

No significant changes in body weight were observed in all DEHP treatment groups except DEHP-40 group when compared to their negative (ORX) and positive group (ORX+ TP), respectively. However, all of the IOO groups shown significant decreases when compared to their DEHP negative (DEHP-40 and DEHP-400) and positive group groups (DEHP-40+TP and DEHP-400+TP), respectively. In IOS groups, significant changes were found in TP groups (IOS$40+\mathrm{TP}$ and IOS-400+TP) compared to their DEHP negative and positive groups, respectively. There were no significant changes in ATEC groups compared to control groups.

\section{2) Weights of $L A B C$}

Like body weight changes, no significant changes in LABC weight were observed in all DEHP treatment groups except DEHP-40 group. In DEHP groups, high dose treatment exhibited significant weight gains compared to the low dose treatment. Significant decreases in LABC weight were observed in all IOO treatment groups and all IOS treatment groups except IOS-400 group compared to DEHP control groups. In ATEC groups, significant de- creases were observed in TP cotreated groups (ATEC40+TP and ATEC-400+TP).

\section{3) Weights of glans penis}

Significant decreases in glans penis weight were observed in low dose DEHP treatment groups (DEHP-40 and DEHP-40+TP) when compared to their control groups. In DEHP groups, high dose treatment exhibited significant weight gains compared to the low dose treatment. All replacement candidate groups exerted significant decreases compared to their DEHP negative and positive groups, respectively.

\section{4) Weights of paired Cowper's glands}

No significant decrease in Cowper's gland weight was observed in all DEHP groups except DEHP-40 group which exerted significant decrement. There was no significant change in all replacement candidate groups when compared to their DEHP negative and positive groups.

\section{5) Weights of seminal vesicle}

No significant change was observed in DEHP groups. There were significant decreases in IOO-40 and IOO-400 groups when compared to their DEHP negative groups. There were significant decreases in IOS+TP cotreated groups and IOS-400 group when compared to their DEHP positive groups and DEHP negative group, respectively. All ATEC group shown no significant change.

\section{6) Weights of ventral prostate}

No significant change in ventral prostate weight was observed in all the DEHP groups except DEHP-40 group when compared to the control groups. No significant change in ventral prostate weight was observed in all the IOO groups except IOO-400+TP group when compared to the DEHP positive groups and DEHP negative group, respectively. There were significant decreases in IOS with TP cotreated groups when compared to the DEHP positive 
Hershberger Assays for Di-2-ethylhexyl Phthalate and Its Substitute Candidates

Table 2. Effects of orally administered di-2-ethylhexyl phthalate (DEHP), 2-ethylhexyl oleate (IOO), 2-ethylhexyl stearate (IOS) and acetyl triethyl citrate (ATEC) and subcutaneously administered testosterone propionate (TP) on body and androgen-sensitive organ weights of castrated rats

\begin{tabular}{|c|c|c|c|c|c|c|}
\hline Treatment $^{\mathrm{a}}$ & $\begin{array}{c}\text { Body } \\
\text { Weight (g) }\end{array}$ & $\begin{array}{l}\text { LABC } \\
(\mathrm{mg})\end{array}$ & $\begin{array}{l}\text { Penis } \\
(\mathrm{mg})\end{array}$ & $\begin{array}{c}\text { Cowper's } \\
\text { glands (mg) }\end{array}$ & $\begin{array}{l}\text { S.V. } \\
(\mathrm{mg})\end{array}$ & $\begin{array}{c}\text { Ventral prostate } \\
(\mathrm{mg})\end{array}$ \\
\hline INT & $301.6 \pm 7.6$ & $736.7 \pm 29.4$ & $271.3 \pm 12.5$ & $26.2 \pm 2.3$ & $193.4 \pm 9.7$ & $146.0 \pm 9.8$ \\
\hline ORX & $281.3 \pm 7.7$ & $301.4 \pm 52.3$ & $158.3 \pm 11.1$ & $5.4 \pm 0.9$ & $28.0 \pm 3.8$ & $29.3 \pm 9.9$ \\
\hline $\mathrm{ORX}+\mathrm{TP}$ & $313.2 \pm 6.3$ & $671.5 \pm 49.0$ & $266.2 \pm 12.8$ & $18.2 \pm 0.8$ & $170.3 \pm 12.5$ & $91.5 \pm 9.7$ \\
\hline DEHP-40 & $276.2 \pm 6.1^{*}$ & $193.7 \pm 17.1$ & $98.3 \pm 2.4^{* * *}$ & $2.3 \pm 0.2^{* * *}$ & $22.4 \pm 1.3$ & $8.5 \pm 0.5^{*}$ \\
\hline DEHP-40+TP & $310.3 \pm 5.2$ & $548.8 \pm 27.5$ & $192.5 \pm 17.1^{\dagger \dagger}$ & $16.6 \pm 1.1$ & $163.9 \pm 8.0$ & $75.6 \pm 4.1$ \\
\hline DEHP-400 & $302.8 \pm 8.7$ & $245.1 \pm 10.5$ & $153.4 \pm 7.5$ & $5.9 \pm 1.2$ & $28.5 \pm 4.0$ & $21.3 \pm 4.7$ \\
\hline DEHP-400+TP & $305.2 \pm 15.9$ & $619.1 \pm 22.0$ & $255.5 \pm 19.3$ & $17.8 \pm 2.5$ & $165.5 \pm 15.4$ & $86.2 \pm 5.5$ \\
\hline IOO-40 & $252.3 \pm 7.1^{*}$ & $153.6 \pm 6.2 \mathrm{z}^{\star}$ & $112.8 \pm 3.0^{* *}$ & $2.6 \pm 0.2^{* *}$ & $16.8 \pm 0.9^{* *}$ & $7.8 \pm 0.5^{*}$ \\
\hline IOO-40+TP & $250.8 \pm 4.7^{\dagger \dagger \dagger}$ & $520.8 \pm 16.7^{\dagger}$ & $218.6 \pm 2.9^{\dagger \dagger}$ & $17.7 \pm 0.9$ & $172.3 \pm 10.4$ & $79.9 \pm 3.0$ \\
\hline IOO-400 & $216.6 \pm 17.9^{* *}$ & $143.8 \pm 12.2^{*}$ & $111.3 \pm 3.2^{* *}$ & $3.2 \pm 0.3^{*}$ & $17.7 \pm 1.0^{*}$ & $7.0 \pm 0.4$ \\
\hline IOO-400+TP & $244.2 \pm 6.6^{\dagger+\dagger}$ & $489.8 \pm 18.4^{\dagger}$ & $199.2 \pm 7.3^{\dagger \dagger}$ & $20.3 \pm 1.4$ & $137.2 \pm 9.5$ & $61.5 \pm 6.2^{\dagger}$ \\
\hline IOS-40 & $265.0 \pm 8.7$ & $163.3 \pm 9.8^{*}$ & $116.9 \pm 5.0^{*}$ & $2.8 \pm 0.2^{* *}$ & $20.4 \pm 1.6$ & $8.4 \pm 0.7$ \\
\hline IOS-40+TP & $281.8 \pm 7.0^{\dagger \dagger}$ & $451.7 \pm 12.9^{\dagger}$ & $217.4 \pm 12.2^{\dagger}$ & $18.1 \pm 0.9$ & $121.6 \pm 5.3^{\dagger \dagger}$ & $58.5 \pm 2.9^{* \dagger \dagger}$ \\
\hline IOS-400 & $274.4 \pm 11.5$ & $210.8 \pm 18.9$ & $138.9 \pm 8.5^{* *}$ & $4.7 \pm 0.7^{\star}$ & $25.3 \pm 1.3^{* * *}$ & $12.4 \pm 1.7$ \\
\hline IOS-400+TP & $286.3 \pm 3.1^{\dagger \dagger}$ & $460.0 \pm 22.8^{\dagger \dagger}$ & $206.1 \pm 6.0^{\dagger \dagger}$ & $17.7 \pm 1.8$ & $138.3 \pm 6.9^{\dagger}$ & $63.9 \pm 2.3^{\dagger}$ \\
\hline ATEC-40 & $287.5 \pm 5.7$ & $209.3 \pm 10.1$ & $123.0 \pm 4.5^{*}$ & $3.8 \pm 0.4$ & $26.0 \pm 1.4$ & $11.5 \pm 0.9$ \\
\hline ATEC-40+TP & $313.9 \pm 10.8$ & $539.2 \pm 21.2^{\dagger}$ & $218.8 \pm 10.1^{\dagger}$ & $17.1 \pm 0.8$ & $159.6 \pm 9.2$ & $80.4 \pm 2.2$ \\
\hline ATEC-400 & $287.9 \pm 9.1$ & $192.4 \pm 6.9^{*}$ & $114.6 \pm 7.0^{* *}$ & $2.2 \pm 0.2^{* * *}$ & $22.7 \pm 1.8$ & $10.9 \pm 1.2$ \\
\hline ATEC-400+TP & $309.0 \pm 3.2$ & $529.3 \pm 24.7^{\dagger}$ & $209.7 \pm 13.0^{\dagger}$ & $18.3 \pm 1.3^{\dagger}$ & $154.3 \pm 10.9$ & $78.2 \pm 3.1$ \\
\hline
\end{tabular}

\footnotetext{
${ }^{\text {a }}$ Immature castrated rats were administered with TP by subcutaneous injection for 10 days. One day after the final treatment, the accessory sex organs were removed carefully and weighed separately.

Symbols ${ }^{*}{ }^{* *}$ and ${ }^{* * *}$ meant significant different in comparison between ORX group and test material group. ${ }^{*} p<0.05$; ${ }^{* *} p<0.01 ;{ }^{* * *} p<0.001$.

Symbols ${ }^{\dagger},{ }^{\dagger \dagger}$ and ${ }^{\dagger+\dagger}$ meant significant different in comparison between ORX+TP group and test material group. ${ }^{\dagger} p<0.05 ;{ }^{\dagger+} p<0.01 ;{ }^{\dagger \dagger} p<0.001$.
}

INT, intact; LABC, levator ani-bulbocavernosus; S.V. seminal vesicle; ORX, orchidectomy. 
groups. No significant changes were found in all ATEC groups.

\section{Effects on androgen-independent organs}

The effects of DEHP and replacement candidates on changes in optional organs (kidney, adrenal glands, spleen and liver) weights in castrated male rats are shown in Table 3.

\section{1) Kidney weights}

Significant increases in kidney weight were observed in DEHP 40 and DEHP 400 groups when compared to their negative controls (ORX group). However, comparison between DEHP positive control groups (DEHP-40 and DEHP$400+\mathrm{TP}$, respectively) and $\mathrm{IOO}+\mathrm{TP}$ cotreated groups exerted significant decreases. Similarly, IOS + TP cotreated groups shown significant decreases compared to DEHP+ TP groups. Significant increases were observed in ATEC groups when compared to control (ORX group), as shown in DEHP groups.

\section{2) Adrenal glands weights}

There was no significant change in DEHP groups except DEHP-40+TP group which exerted significant decrease compared to control groups $(\mathrm{ORX}+\mathrm{TP})$. IOO+TP cotreated groups shown significant decreases compared to the DEHP positive groups, DEHP-40+TP and DEHP-400+TP, respectively. There were no significant changes in IOS groups and ATEC groups, except IOS-400 and ATC-40+TP which shown significant decreases.

\section{3) Spleen weights}

No significant change in spleen weight was observed in all treatment groups except IOO-400 group.

\section{4) Liver weights}

No significant change in adrenal glands weight was observed in all DEHP groups. There were significant decreases in IOO groups except IOO-400+TP group. In IOS groups, significant decrease was observed only in IOS-40 group compared to control group. In ATEC groups, no significant change was found.

\section{DISCUSSION}

DEHP is well known to have potential hazards on animal and human health, such as reproductive and developmental toxicity via disruption of the endocrine system (Zarean et al., 2016). As a typical example, DEHP and some other phthalates act as anti-androgens and can cause cryptorchidism in rodents and probably in human. One plausible hypothesis for occurrence of this symptom is an exposure to a chemical (or a mixture of chemicals) with anti-androgenic or estrogenic properties before and during the critical timing of external genital differentiation (Toppari et al., 2006). Indeed, maternal DEHP exposure could disrupt fetal testicular development (Shirota et al., 2005) and testosterone production (Parks et al., 2000; Howdeshell et al., 2008), and these findings support the hypothesis.

Since DEHP is the most common phthalate plasticizer in medical devices such as intravenous tubing and bags, special concern has been expressed about leachates of DEHP transported into the patient, especially for those requiring extensive infusions or those who are at the highest risk of developmental abnormalities, e.g. neonates, premature babies, lactating and pregnant women (Tickner et al., 2001; Latini et al., 2010). Therefore, development of plastic products with DEHP alternatives which can ensure safety becomes an urgent issue. Several bio-based plasticizers have been and are being developed as alternatives to phthalates. Among them, a plasticizer based on vegetable oil which is compatible as a primary plasticizer has been developed and is a ready substitute for dioctyl phthalate (Privas et al., 2013). Isosorbide, a BPA replacement, is a non-toxic biodegradable diol derived from bio-based feedstock, and it can be used for preparing thermoplastic starch 
Table 3. Effects of orally administered DEHP, IOO, IOS and ATEC and subcutaneously administered TP on androgen-insensitive organ weights of castrated rats

\begin{tabular}{|c|c|c|c|c|}
\hline Treatment $^{\mathrm{a}}$ & $\begin{array}{l}\text { Kidney } \\
\text { (mg) }\end{array}$ & $\begin{array}{c}\text { Adrenal glands } \\
\text { (mg) }\end{array}$ & $\begin{array}{l}\text { Spleen } \\
(\mathrm{mg})\end{array}$ & $\begin{array}{l}\text { Liver } \\
(\mathrm{g})\end{array}$ \\
\hline INT & $1,150.1 \pm 25.2$ & $25.2 \pm 1.4$ & $740.5 \pm 27.6$ & $12.7 \pm 0.6$ \\
\hline ORX & $931.8 \pm 19.5$ & $28.4 \pm 1.9$ & $699.5 \pm 33.5$ & $12.0 \pm 0.7$ \\
\hline $\mathrm{ORX}+\mathrm{TP}$ & $1,169.8 \pm 13.8$ & $28.2 \pm 1.3$ & $822.8 \pm 56.5$ & $12.1 \pm 0.8$ \\
\hline DEHP-40 & $1,037.9 \pm 19.0^{* * *}$ & $25.7 \pm 0.7$ & $715.8 \pm 54.7$ & $11.1 \pm 0.4$ \\
\hline DEHP-40+TP & $1,190.4 \pm 37.1$ & $22.5 \pm 1.0^{\dagger \dagger}$ & $765.4 \pm 23.1$ & $14.0 \pm 0.7$ \\
\hline DEHP-400 & $1,178.6 \pm 19.1^{* * *}$ & $31.6 \pm 1.2$ & $671.6 \pm 50.6$ & $11.9 \pm 1.4$ \\
\hline DEHP-400+TP & $1,164.4 \pm 32.9$ & $26.8 \pm 1.2$ & $741.7 \pm 61.0$ & $13.1 \pm 1.0$ \\
\hline IOO-40 & $923.7 \pm 51.7$ & $24.8 \pm 0.6$ & $726.8 \pm 19.2$ & $10.4 \pm 0.2^{*}$ \\
\hline $\mathrm{IOO}-40+\mathrm{TP}$ & $991.3 \pm 21.2^{\dagger \dagger \dagger}$ & $23.4 \pm 0.9^{\dagger \dagger}$ & $747.8 \pm 49.2$ & $10.1 \pm 0.3^{\dagger}$ \\
\hline IOO-400 & $880.5 \pm 43.0$ & $26.1 \pm 1.4$ & $561.4 \pm 95.7^{*}$ & $8.3 \pm 1.0^{* *}$ \\
\hline $\mathrm{IOO}-400+\mathrm{TP}$ & $986.6 \pm 20.4^{\dagger+\dagger}$ & $19.5 \pm 1.1^{\dagger \dagger \dagger}$ & $737.1 \pm 53.1$ & $11.3 \pm 0.3$ \\
\hline IOS-40 & $967.3 \pm 15.9$ & $26.4 \pm 1.2$ & $761.3 \pm 37.1$ & $10.1 \pm 0.4^{*}$ \\
\hline IOS-40+TP & $1,064.5 \pm 22.7^{\dagger \dagger \dagger}$ & $27.4 \pm 0.7$ & $821.0 \pm 28.3$ & $11.2 \pm 0.3$ \\
\hline IOS-400 & $978.2 \pm 22.8$ & $31.8 \pm 1.8^{*}$ & $673.8 \pm 35.6$ & $10.7 \pm 0.6$ \\
\hline IOS-400+TP & $1,080.7 \pm 28.3^{\dagger \dagger}$ & $27.2 \pm 0.8$ & $798.6 \pm 64.8$ & $12.1 \pm 0.2$ \\
\hline ATEC-40 & $1,046.8 \pm 20.2^{* * *}$ & $26.5 \pm 1.1$ & $742.9 \pm 28.9$ & $11.4 \pm 0.5$ \\
\hline ATEC-40+TP & $1,158.7 \pm 31.8$ & $21.7 \pm 1.2^{\dagger \dagger}$ & $749.1 \pm 37.2$ & $12.6 \pm 0.7$ \\
\hline ATEC-400 & $1,088.6 \pm 20.8^{* * *}$ & $24.5 \pm 0.8$ & $663.6 \pm 26.3$ & $11.8 \pm 0.4$ \\
\hline ATEC-400+TP & $1,206.8 \pm 10.5^{\dagger}$ & $26.2 \pm 1.4$ & $829.5 \pm 32.7$ & $13.5 \pm 0.4$ \\
\hline
\end{tabular}

${ }^{a}$ Immature castrated rats were administered with TP by subcutaneous injection for 10 days. One day after the final treatment, the accessory sex organs were removed carefully and weighed separately.

Symbols ${ }^{*},{ }^{* *}$ and ${ }^{* * *}$ meant significant different in comparison between ORX group and test material group. ${ }^{*} p<0.05$; ${ }^{* *} p<0.01 ;{ }^{* * *} p<0.001$.

Symbols ${ }^{\dagger}$, ${ }^{\dagger}$ and ${ }^{\dagger+}+$ meant significant different in comparison between ORX group and test material group. ${ }^{\dagger} p<0.05 ;{ }^{\dagger}{ }^{+} p<0.01 ;{ }^{\dagger+}{ }^{\dagger} p<0.001$.

INT, intact; ORX, orchidectomy. 
through a semi-industrial process of extrusion, allowing some technological advantages with respect to classical plasticizers (Battegazzore et al., 2015).

Although there are some biological alternatives on the market, the problem is that they are typically expensive and not coISmpatible as a primary plasticizer. Furthermore, the potential negative effects of the candidates could not be screened accurately and efficiently. For example, there is ample of evidence on the adverse effects of bisphenol-S (BPS), an alternative of BPA (Usman \& Ahmad, 2016; Rosenfeld, 2017). The Hershberger Bioassay is a short-term in vivo screening test using accessory tissues of the male reproductive tract to detect compounds with potential to exert androgenic or antiandrogenic activities (EPA, 2011; OECD, 2008). This assay is relatively convenient and reliable because five androgen-dependent organs, in castrated rats, sensitively respond to chemical with androgenic or antiandrogenic activities as an increase or decrease in absolute weights (Marty \& O'Connor, 2014). We previously evaluated the two BPA substitute candidates whether they have androgenic or antiandrogenic activity using Hershberger assay, and found isosorbide could be a safer candidate while cyclohexanedimethanol exerted more detrimental effects (Kim et al., 2017).

In the present study, we found that all test materials including DEPC did not exhibit any androgenic activity in the Hershberger assay. Rather, antiandrogen-like activities were found in all test materials, and the order of the intensity was ATEC $<$ DEHP $<$ ISO $<$ IOO in 5 androgensensitive organs. There is no available information on the action mechanism of the test materials (i.e. their receptors), so we tentatively concluded the weight reduction effects of these materials are derived from antiandrogen-like activities. Meanwhile, no androgenic or antiandrogenic activities of the test materials were found in the androgen-insensitive organs used in this study. Generally, the adverse effects of ATEC treatment on the accessory sex organs were much less or not present at all when compared to those of DEHP.
So we concluded that ATEC could be a DEHP substitute. IOO treatment brought most severe weight reduction in all of androgen-sensitive organs, so this material should be excluded for further screening of DEHP substitute selection.

\section{ACKNOWLEDGEMENT}

The authors appreciate Mr. Yong Bin Kim for his technical assistance. This study was supported by a grant of National Research Foundation of Korea (NRF-2015M3A9D 7067365).

\section{REFERENCES}

Battegazzore D, Bocchini S, Nicola G, Martini E, Frache A (2015) Isosorbide, a green plasticizer for thermoplastic starch that does not retrogradate. Carbohydr Polym 119:78-84.

Cao XL (2009) Phthalate esters in foods: Sources, occurrence, and analytical methods. Compr Rev Food Sci Food Saf 9:21-43.

EPA (US Environmental Protection Agency) (2011) Endocrine Disruptor Screening Program: Hershberger Assay OCSPP Guideline 890.1400 Standard Evaluation Procedure (SEP). Washington, DC.

Gray LE Jr, Ostby J, Furr J, Price M, Veeramachaneni DN, Parks L (2000) Perinatal exposure to the phthalates DEHP, BBP, and DINP, but not DEP, DMP, or DOTP, alters sexual differentiation of the male rat. Toxicol Sci 58:350-365.

Guo Y, Alomirah H, Cho HS, Minh TB, Mohd MA, Nakata H, Kannan K (2011) Occurrence of phthalate metabolites in human urine from several Asian countries. Environ Sci Technol 45:3138-3144.

Howdeshell KL, Wilson VS, Furr J, Lambright CR, Rider CV, Blystone CR, Hotchkiss AK, Gray LE Jr (2008) A mixture of five phthalate esters inhibits fetal testicular 
testosterone production in the Sprague-Dawley rat in a cumulative, dose-additive manner. Toxicol Sci 105: 153-165.

Huang XF, Li Y, Gu YH, Liu M, Xu Y, Yuan Y, Sun F, Zhang HQ, Shi HJ (2012) The effects of di-(2-ethylhexyl)-phthalate exposure on fertilization and embryonic development in vitro and testicular genomic mutation in vivo. PLoS One 7(11):e50465.

Kay VR, Chambers C, Foster WG (2013) Reproductive and developmental effects of phthalate diesters in females. Crit Rev Toxicol 43:200-219.

Kim HS, Kim YB, Choi D, Cheon YP, Lee SH (2017) Hershberger assays for bisphenol-A and its substitute candidates. Dev Reprod 21:441-448.

Latini G, Ferri M, Chiellini F (2010) Materials degradation in PVC medical devices, DEHP leaching and neonatal outcomes. Curr Med Chem 17:2979-2789.

Lyche JL, Gutleb AC, Bergman A, Eriksen GS, Murk AJ, Ropstad E, Saunders M, Skaare JU (2009) Reproductive and developmental toxicity of phthalates. J Toxicol Environ Health B Crit Rev 12:225-249.

Martino-Andrade AJ, Chahoud I (2010) Reproductive toxicity of phthalate esters. Mol Nutr Food Res 54:148157.

Marty MS, O'Connor JC (2014) Key learnings from the endocrine disruptor screening program (EDSP) tier 1 rodent uterotrophic and Hershberger assays. Birth Defects Res B Dev Reprod Toxicol 101:63-79.

Meeker JD, Sathyanarayana S, Swan SH (2008) Phthalates and other additives in plastics: human exposure and associated health outcomes. Philos Trans R Soc Lond B Biol Sci 364:2097-2113.

OECD (2008) Draft OECD Guideline for the Testing of
Chemicals - The Hershberger Bioassay in Rats: A Shortterm Test for (Anti)Androgenic Properties.

Parks LG, Ostby JS, Lambright CR, Abbott BD, Klinefelter GR, Barlow NJ, Gray LE Jr (2000) The plasticizer diethylhexyl phthalate induces malformations by decreasing fetal testosterone synthesis during sexual differentiation in the male rat. Toxicol Sci 58:339-349.

Privas E, Leroux F, Navard P (2013) Preparation and properties of blends composed of lignosulfonated layered double hydroxide/plasticized starch and thermoplastics. Carbohydr Polym 96:91-100.

Rosenfeld CS (2017) Neuroendocrine disruption in animal models due to exposure to bisphenol A analogues. Front Neuroendocrinol 47:123-133.

Shirota M, Saito Y, Imai K, Horiuchi S, Yoshimura S, Sato M, Nagao T, Ono H, Katoh M (2005) Influence of di(2-ethylhexyl) phthalate on fetal testicular development by oral administration to pregnant rats. J Toxicol Sci 30:175-194

Tickner JA, Schettler T, Guidotti T, McCally M, Rossi M (2001) Health risks posed by use of di-2-ethylhexyl phthalate (DEHP) in PVC medical devices: a critical review. Am J Ind Med 39:100-111.

Toppari J, Virtanen H, Skakkebaek NE, Main KM (2006) Environmental effects on hormonal regulation of testicular descent. J Steroid Biochem Mol Biol 102:184186.

Usman A, Ahmad M (2016) From BPA to its analogues: Is it a safe journey? Chemosphere 158:131-142.

Zarean M, Keikha M, Poursafa P, Khalighinejad P, Amin M, Kelishadi R (2016) A systematic review on the adverse health effects of di-2-ethylhexyl phthalate. Environ Sci Pollut Res Int 23:24642-24693. 